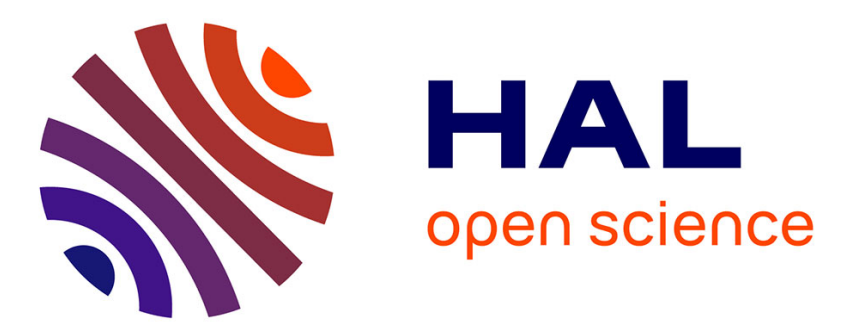

\title{
An optimization framework for the joint routing and scheduling in wireless mesh networks
}

Christelle Molle, Fabrice Peix, Hervé Rivano

\section{To cite this version:}

Christelle Molle, Fabrice Peix, Hervé Rivano. An optimization framework for the joint routing and scheduling in wireless mesh networks. Proc. 19th IEEE International Symposium on Personal, Indoor and Mobile Radio Communications (PIMRC'08), Sep 2008, Cannes, France. pp.O84-2. hal-00429829

\section{HAL Id: hal-00429829 \\ https://hal.science/hal-00429829}

Submitted on 4 Nov 2009

HAL is a multi-disciplinary open access archive for the deposit and dissemination of scientific research documents, whether they are published or not. The documents may come from teaching and research institutions in France or abroad, or from public or private research centers.
L'archive ouverte pluridisciplinaire HAL, est destinée au dépôt et à la diffusion de documents scientifiques de niveau recherche, publiés ou non, émanant des établissements d'enseignement et de recherche français ou étrangers, des laboratoires publics ou privés. 


\title{
An optimization framework for the joint routing and scheduling in wireless mesh networks
}

\author{
Christelle Molle, Fabrice Peix, Hervé Rivano \\ MascotTE project, I3S(CNRS-UnSA)/InRIA, Sophia Antipolis, France. \\ E-mail: \{christelle.molle, fabrice.peix, herve.rivano\}@sophia.inria.fr.
}

\begin{abstract}
In this paper, we address the problem of computing the transport capacity of Wireless Mesh Networks dedicated to Internet access. Routing and transmission scheduling have a major impact on the capacity provided to the clients. A crosslayer optimization of these problems allows the routing to take into account contentions due to radio interferences. We develop exact linear programs and provide an efficient column generation process computing a relaxation of the problem. It allows to work around the combinatoric of simultaneously achievable transmissions, hence computing solutions on large networks. Our approach is validated through extensive simulations. Evolution of the capacity of a mesh network with its parameters, as well as the algorithmic complexity are then discussed. We conjecture that the problem can be solved in polynomial time and that the gateway placement problem is only subject to localized constraints.

Index Terms-Wireless Mesh Networks, Capacity, Routing, Scheduling, Linear Programming, Column Generation.
\end{abstract}

\section{INTRODUCTION}

Wireless mesh networks (WMNs) are cost-effective solutions for ubiquitous high-speed services [1]. They are selforganized networks with a fixed infrastructure of wireless mesh routers interconnected to provide Internet access to mobile network users. This infrastructure, forming a wireless backhaul network, is integrated with Internet by special routers called mesh gateways (Fig. I).

The problem of providing full optimization frameworks for WMNs has received significant attention lately. A very important requirement for the network performance evaluation is to maximize its capacity, i.e. the throughput offered to each flow. However, one of the major issue of wireless networks is the capacity reduction due to radio interferences [2], [3]. Several studies have pointed out a common reduction phenomenon in ad-hoc networks [4], [5], [6]. However, WMN gathers traffic in the gateways, leading to bottlenecks around them and a more constrained available capacity per node [7].

The goal of this paper is to provide models and frameworks determining the optimal theoretical capacity of a WMN. Capacity evaluation with a cross-layer approach is useful to improve the performance of routing protocols and MAC layers. Routing efficiency depends on the problem of allocating the resources of the OSI physical and data link layers. Indeed, interferences between link transmissions clearly impact the routing used to satisfy traffic demands. Similarly, traffic routing determines the traffic flow requirements on each link, according to the achievable capacity of the links.

In order to maximize the capacity, one has to determine an optimal allocation of the shared resources, in particular

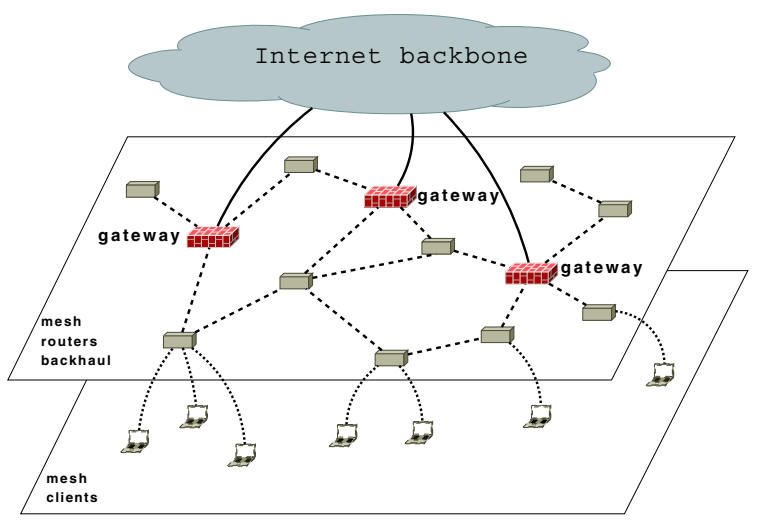

Fig. 1. A WMN topology: mobile clients access Internet through a multi-hop wireless backhaul network of routers and gateways.

the radio channel available. This allocation is constrained by interferences and contentions around a transmitting node: simultaneous transmissions must be apart from several hops in the network. Contending links share a common channel using a time multiplexing process. Optimal ressources allocation can therefore be related to a link scheduling in order to route a given arbitrary traffic distribution at maximum rate [8].

The strongest hypothesis of our work is to consider a synchronous network and a time period divided into slots of equal duration. Each link is assigned a set of slots during which it is activated. Each slot is assigned to a set of pairwise non interfering links, leading to a feasible transmission schedule. These sets are called rounds. As far as the set of all possible rounds is exponential, finding an optimal schedule using simple methods is only tractable for small-sized networks. Optimization-based approaches trying to reduce the size of the space of rounds have been proposed [9] in conjunction with approximation algorithms [10].

Column generation is a prominent technique to cope with large-scale integer programs [11]. When the number of variables is exponential, it provides a decomposition of the problem into different parts. The master program solves the problem for a subset of variables associated to combinatorial structures. These structures are generated by sub-programs called auxiliary programs, selecting new structures that improve the master solution. This technique has already been used to solve the problem where multi-routes, as well as router to multigateway traffic, are forbidden [12], [13]. Nevertheless, multi- 
path routing increases the end-to-end network throughput [14], and a multi-gateway association results in better capacity and fairness in the network [15].

The contribution of this work is to present two exact formulations of the routing and scheduling problem in wireless mesh networks (Section III), and compute optimal solutions by using a column generation process where both paths and rounds are generated in auxiliary programs (Section IV). This opens the door to more realistic generations operated, as for an example, by event-driven network simulations. This process is validated through extensive simulations. Capacity evolution and algorithmic complexity of the problem are discussed (Section V). In the next section, we present the optimization problem of routing and scheduling transmissions in a wireless mesh network.

\section{Problem Definition and Assumptions}

The fixed infrastructure of the WMN is modeled by a directed graph $G=(V, E)$ of $N$ nodes representing mesh points. Each device has a single interface allowing to send or receive packets on the only channel available. This channel is therefore shared between all the nodes. The set of vertices $V$ is decomposed into two non-intersecting subsets: $V_{g}$ is the set of mesh gateways and $V_{r}$ the set of mesh routers that do not interact directly with Internet. Each router $r$ of $V_{r}$ has a demand $d_{r}$ to send to the gateways, corresponding to the aggregated traffic of the mobile clients connected to it.

In the following, the network is assumed to be synchronous. We focus on the steady state of the network, which is therefore periodic with a period duration $T$. The communication protocol is a slotted TDMA multiplexing. Computing an optimal allocation of the slots to the links taking radio interferences into account refers to the throughput provided to each router. This throughput corresponds to the ratio of the volume of data sent during a period over $T$, the duration of the period.

To ensure transmissions, during a time slot, links activated have to be pairwise interference-free. The structure of these sets thus depends on both the MAC layer of the network and the radio interferences. The MAC layer CSMA/CA implemented in IEEE 802.11 uses RTS/CTS messages for a pair of nodes to reserve the channel and transmit. Many interference models have been developped on top of CSMA/CA. They are called binary models, meaning that two nodes can either always or never transmit simultaneously unless they communicate together [16] . These binary models can be represented by a conflict graph $G_{c}=\left(V_{c}, E_{c}\right)$, where a node in $V_{c}$ corresponds to a link of $E$ in $G$, and a link between two nodes of $V_{c}$ exists if and only if the corresponding links are interfering in $G$.

Let $\mathcal{I}(e)$ be the set of links interfering with $e$ of $E$ in the network. Given a binary interference model, a set $s \subseteq E$ in $G$ is called a round and contains pairwise non-interfering transmissions that can be active at the same time: $s \subseteq E$, $\forall e_{1}, e_{2} \in s, e_{1} \notin \mathcal{I}\left(e_{2}\right)$ and $e_{2} \notin \mathcal{I}\left(e_{1}\right)$. In the conflict graph, a round actually corresponds to an independent set, or stable set. Recall that an independent set of a graph is a subset of

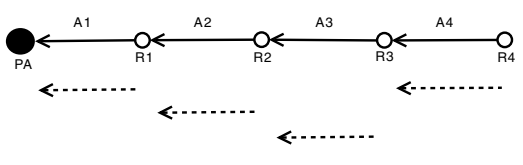

(a) $G=(V, E)$

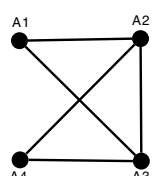

(b) $\mathrm{Gc}=(\mathrm{Vc}, \mathrm{Ec})$
Fig. 2. Distance-2 interference model: (a) Topology graph, (b) Conflict graph. Sets of compatible transmissions $\{A 1, A 4\},\{A 2\},\{A 3\}$ correspond to independent sets of the conflict graph.

nodes such that there is no link between them. This is depicted in Fig. 2 using a distance-2 binary interference model in which two transmissions do not interfere if there are at least two hops between them. For instance, links $A_{1}$ and $A_{4}$ are separated by $A_{2}$ and $A_{3}$, they can thus be in the same round. Similarly, one can see that $\left\{A_{1}, A_{4}\right\}$ is an independent set of $G_{c}$ since the corresponding nodes are not adjacent.

The scheduling problem consists in determining the round activations during a time period. To ensure that data are sent from the routers to the gateways, all links of a path between the sources and the destinations have to be activated during the time period. Then, an active path with $\lambda$ units of flow on its most constrained link during $[1, T]$ has a flow rate of $\lambda / T$ in permanent regime. Routing at maximum rate then determines a set of paths between the sources and destinations whose links can be scheduled in a minimum period of time.

\section{JOINT ROUTING AND SCHEDULING OPTIMIZATIONS}

We consider the problem of simultaneously (i) computing paths joining mesh routers to mesh gateways and (ii) selecting rounds in order to route the traffic along these paths with maximum throughput.

\section{A. Node/Arc Formulation}

This formulation of the joint routing and scheduling problem, called node/arc formulation, is based on the multiflow problem constraints, with additional scheduling constraints.

Multiflow computes paths between mesh routers (MRs) and gateways (MGs). Multiflow constraints express the injection of traffic $d_{r}$ by MR $r$ into the network that is forwarded without loss by other MRs until its extraction by the MGs. Let $f_{e}^{r}$ be the flow sent by $r$ going through link $e$. Then the capacity of link $e$ is shared by the flows $f_{e}^{r}$ going through it.

Scheduling is defined with binary variables $a_{e}^{t}$ which indicate if a given link $e$ is activated during slot $t$ of the network period, and $y_{t}$ which indicate if at least one link is activated at time $t$. Data are normalized such that a link has a unitary capacity during a slot of the period. Constraints express the contentions between two interfering transmissions. The network period length equals the number of used slots, i.e. slots during which at least one link is selected. A bound on this length is fixed, $T_{\max }$, and the set of used slots in the frame $\left[1, T_{\max }\right]$ is minimized.

This leads to the following integer linear program, where $\Gamma^{+}(v)$ (respectively $\Gamma^{-}(v)$ ) denotes the set of outgoing arcs (respectively ingoing arcs) of node $v$. 


$$
\begin{gathered}
\min \sum_{t \leqslant T_{\max }} y_{t} \\
\sum_{e \in \Gamma^{+}(v)} f_{e}^{r}-\sum_{e \in \Gamma^{-}(v)} f_{e}^{r}=0, \forall r \in V_{r}, v \in V_{r} \backslash\{r\} \\
\sum_{g \in V_{g}} \sum_{e \in \Gamma^{-}(g)} f_{e}^{r}=\sum_{e \in \Gamma^{+}(r)} f_{e}^{r}=d_{r}, \forall r \in V_{r} \\
a_{e}^{t}+a_{e^{\prime}}^{t} \leqslant 1, \forall e \in E, e^{\prime} \in \mathcal{I}(e), t \leqslant T_{\max } \\
\sum_{r \in V_{r}} f_{e}^{r} \leqslant \sum_{t \leqslant T_{\max }} a_{e}^{t}, \forall e \in E \\
a_{e}^{t} \leqslant y_{t}, \forall e \in E, t \leqslant T_{\max }
\end{gathered}
$$

Objective (1) corresponds to a flow rate maximization in a steady state of the network: if $\lambda$ units of flow are sent from a router to a gateway in $T$ units of time, then the flow rate is $\lambda / T$ in steady state. Constraints (2)-(3) describe the flow conservation: a MR $r$ sends $d_{r}$ units of flow, forwarded by other MRs, until they are received by MGs. Constraints (4) ensure the round construction at every time slot $t$ : a link $e$ cannot be active in conjunction with a link $e^{\prime} \in \mathcal{I}(e)$, following the definition of the interference set $\mathcal{I}(e)$ presented above. Constraints (5) link the routing and the scheduling: the total flow on a link cannot exceed its global capacity on the time period, corresponding to the number of slots on which the link is activated. Constraints (6) compute the set of slots effectively used during $\left[1, T_{\max }\right]$, that is the slots during which at least one link is activated.

This integer linear formulation has a lot of binary variables and generates thousands of constraints. This combinatorial hardness makes the problem intractable for topologies with more than twenty nodes. Some optimal results have been found for the joint routing and scheduling problem with a dual objective function on small topologies in [17].

In order to tackle larger topologies, we introduce another approach using a relaxation of the problem.

\section{B. Path/Round Formulation}

A continuous relaxation of the node/arc formulation would be irrelevant since the round structure is deeply dependent on the binary form of the $a_{e}^{t}$ variables. As for an example, one could set all $a_{e}^{t}$ to $1 / 2$. Constraints (4) would always be satisfied but this feasible solution is useless to build efficient rounds. This is a very generic assessment where exclusions are expressed by binary variables, like in colouring problems.

We therefore provide another formulation of the problem, where contentions are expressed by variables on combinatorial objects instead of constraints on binary variables.

One can notice that a complete link scheduling is not necessary. Indeed, permuting slots does not change the solution cost. Getting the set of activated rounds in an optimal solution is enough to construct an optimal scheduling, assigning a frame to each round in an arbitrary order. Hence the problem can be reduced to a round weighting problem [10]. By allowing this weighting to be fractional instead of integer, one can keep the fundamental structure of the original problem in an effective relaxed scheme.

Each round $s$ can be selected $a_{s}$ times during the network period. In the relaxed problem, $a_{s}$ becomes the duration of the round selection. Only one round can be selected at a time, hence the sum of the round activation durations equals the period length, which is to be minimized. Recall that routing at maximum rate in permanent regime is to select paths whose links can be scheduled in a minimum period of time. The capacity of a given link is then given by the sum of the activation durations over the rounds containing the link.

Similarly, one can introduce a set of paths instead of the multiflow constraints on links. The routing problem thus becomes a selection of the set of paths carrying the flows. Let $\mathcal{P}_{r}$ be the set of paths going from router $r$ to the gateways and $f_{p}$ the amount of flow on path $p \in \mathcal{P}_{r} . \mathcal{P}=\cup_{r} \mathcal{P}_{r}$ denotes the set of all paths, and $\mathcal{S}$ the set of the rounds.

The routing and scheduling problem is written as follows.

$$
\begin{aligned}
& \min \sum_{s \in \mathcal{S}} a_{s} \\
\sum_{p \in \mathcal{P}, p \ni e} f_{p} & \leqslant \sum_{s \in \mathcal{S}, s \ni e} a_{s}, \forall e \in E \\
\sum_{p \in \mathcal{P}_{r}} f_{p} & =d_{r}, \forall r \in V_{r}
\end{aligned}
$$

In this new formulation, the number of constraints is strongly reduced because flow conservation and round construction constraints are no longer necessary; it is hard coded into the structure of the combinatorial variables. Only capacity constraints (8) and demand constraints (9) are expressed.

Conversely, the number of variables is now exponential. Indeed, it exists an exponential number of paths between a pair (source, destination) in the graph, as well as possible rounds. Therefore this formulation cannot be manipulated with the whole set of variables when the size of the network grows.

Column generation is made for coping with large sets of variables, thus avoiding the enumeration of all the possible variables as described below.

\section{COLUMn Generation}

Column generation is a primal/dual process for solving linear programs with an exponential set of variables.

The scheme is to first solve the continuous relaxation of the path/round linear program with restricted sets of paths $\mathcal{P}_{0}$ and rounds $\mathcal{S}_{0}$. This so-called master program is thus fastly solved. If there exists a feasible solution of this restricted master problem, current optimal primal and dual solutions are obtained. The initial sets have to be carefully chosen so that an initial feasible solution exists. Fortunately, this is the case of the obvious sets $\mathcal{P}_{0}$ containing a shortest path from each router to a gateway, and $\mathcal{S}_{0}=\{\{e\}, e \in E\}$.

Considering a solution, optimal for restricted sets of paths and rounds, the resulting optimal dual variables define weight functions on the nodes and links of $G$. They are given as 
parameters of auxiliary programs which seek paths or rounds violating the dual constraints as detailed below. If such paths or rounds exist, they correspond to new columns that can be added to the master program. The separation theorem claims that solving again the master produces a better solution [18]. The process loops until no such column exists. When reaching this state, the theory of duality claims that both the primal and the dual are optimized.

\section{A. Dual Formulation}

In our case, the dual of the master problem involves two constraints corresponding to the path and round variables $f_{p}$ and $a_{s}$. Introducing the dual variables $y_{e}, e \in E$ for links associated with constraint (8), and $x_{u}, u \in V_{r}$ for mesh routers associated with constraint (9), the dual formulation consists in maximizing a volume-like mesure of the network, $\sum_{r \in V_{r}} d_{r} x_{r}$, under the following path length and round weight constraints:

$$
\begin{aligned}
\sum_{e \in p} y_{e} & \geqslant x_{\mathcal{O}(p)}, \forall p \in \mathcal{P} \\
1 & \geqslant \sum_{e \in s} y_{e}, \forall s \in \mathcal{S}
\end{aligned}
$$

where $\mathcal{O}(p)$ denotes the source node of path $p$.

The problem is now to determine if there exists new paths and rounds that violate these constraints and make the objective function of the master program increase.

\section{B. Auxiliary Programs}

The column generation process we use for our optimization problem involves two auxiliary programs. Given the dual link variables, the first subproblem aims at finding a weighted path that violates the dual constraint (10), that is, a path with associated weight lower than the dual variable associated to its source node. Shortest path computation from the routers to the gateways always gives either the most violated constraint, hence a good candidate column to be added, or a proof that all constraints are fit. If relevant, the path is added in the current set of variables of the master program.

Similarly, with the same link weighting, the second auxiliary program computes a set of compatible transmissions with total weight greater than 1, i.e. violating constraint (11). Computing a maximum weighted independent set of the conflict graph gives a candidate or certify that no constraint is violated.

Note that computing maximum independent sets is $\mathrm{NP}$ hard in general graphs. However, simulations give a hint that complexity is not a major issue in the specific case of the weight functions induced by the dual of a concentration flow on the gateways.

\section{Simulations}

The two formulations and the centralized column generation process have been implemented using the MASCOPT library developed by team members, and ILOG CPLEX solver.

Tests have been realized on many instances like grids

\begin{tabular}{|c|c|c|c|}
\hline Network size & $\mathrm{Nb}$ of gtws & \multicolumn{2}{|c|}{ Resolution time (ms) } \\
\hline & & node/arc & path/round \\
\hline 10 & 1 & 4271 & 448 \\
\hline 10 & 3 & 4069 & 512 \\
\hline 20 & 1 & 107515 & 1147 \\
\hline 20 & 2 & 103774 & 1386 \\
\hline 20 & 5 & 105485 & 1055 \\
\hline 30 & 1 & 713571 & 6684 \\
\hline 50 & 1 & 20539940 & 8513 \\
\hline 70 & 1 & $\ldots$ & 74516 \\
\hline 100 & 1 & $\ldots$ & 448912 \\
\hline
\end{tabular}
(Manhattan like networks) and general mesh topologies. In the
TABLE I

COMPARISON OF THE FORMULATIONS. TIME RESOLUTION IS GREATLY IMPROVED BY THE PATH/ROUND FORMULATION.

following we present our results on a set of random instances based on $n$ points Poisson process. We have generated topologies with 10 to 100 nodes, on which is either routed a uniform traffic of value 1, or a random i.i.d. traffic between 1 and 20 for each source node. We use the classical distance-2 interference model presented in Section II. For each topology, we have tested with 1 to $n / \bar{d}$ uniformly spread gateways, where $\bar{d}$ is the mean node degree. In the last case, gateways only communicate with their neighbors, in average. These results show common characteristics with tests on other instances.

Since the node/arc formulation computes a full slotted link scheduling over time, while the path/round formulation computes a relaxed fractional round weighting, the optimal period length of the latter is a lower bound of the former. As a matter of fact, in any of our test instances, the relaxed period equals the optimal integral one (up to rounding it up). This validate both our approach and the formulation. Preliminary combinatorial results seem to prove this observation up to a small constant in any case.

a) Complexity issues: Resolution times for the node/arc and the path/round formulations are presented in Table I. The path/round formulation computes results for big topologies in a few seconds, while the node/arc formulation cannot handle networks larger than few tens of nodes. A more detailed look on the results show that generating the rounds is sub-linear in the network size, but almost linear in the gateways density.

In fact, we conjecture that the problem is polynomial: inside a bounded size contention area surrounding each gateway, one would need to carefully optimize the scheduling. Outside, the linear program would be so loosely constrained that the solutions of fast non-optimal protocol would fit.

In the following, we present our results on the transport capacity of the network.

b) Capacity vs network size: In a data gathering environment with one gateway, previous works have shown that the per-node capacity is decreasing linearly with the network size [10]. Figure 3 confirms this phenomenon with several gateways and networks bigger than 20 nodes.

c) Capacity us gateway density: Linear dependency between capacity and gateway density corresponds to an obvious upper bound, but interferences between gateways could degrade performances. To study this phenomenon, we have 


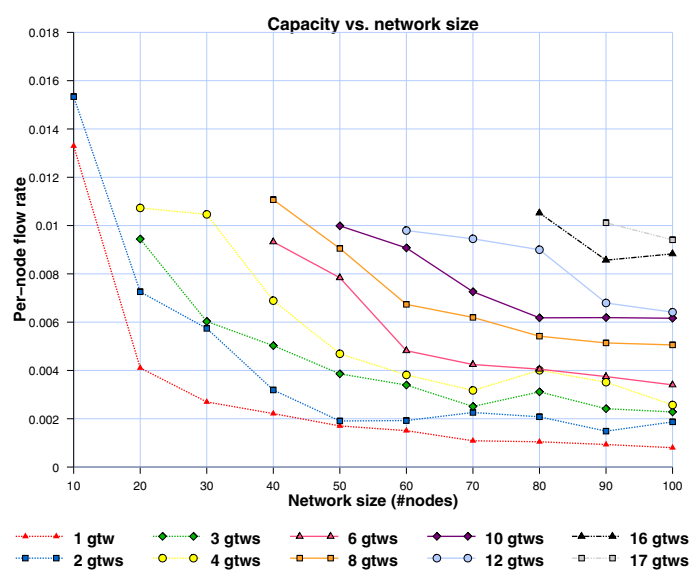

Fig. 3. Capacity evolution vs size of the network.

generated different random gateway placements for each topology. Figure 4 presents the mean gain obtained by adding new gateways. Values are normalized by single gateway rate.

Results show a linear gain in the number of gateways with a gradient slightly lower than 1 . The placement of the gateway is expected to have a strong impact on the network performances [17]. Simulations moderate this assertion. On one hand, when gateways are too close from each other, simulations show that they actually act as only one: interferences on the links around each gateway merge them and prevent a simultaneous traffic gathering. On the other hand, with a correct placement, even non optimized, wireless mesh networks act efficiently.

We conjecture that there exists a minimum interspace between the gateways, that is obviously necessary, but also sufficient, to an efficient traffic flow. This thickness is expected to be explicitely computed on regular topologies, and estimated with binary interference models.

\section{CONCLUSION}

In this work, we have presented a cross-layer optimization framework for the joint routing and scheduling problem in Internet-providing wireless mesh networks. This framework is

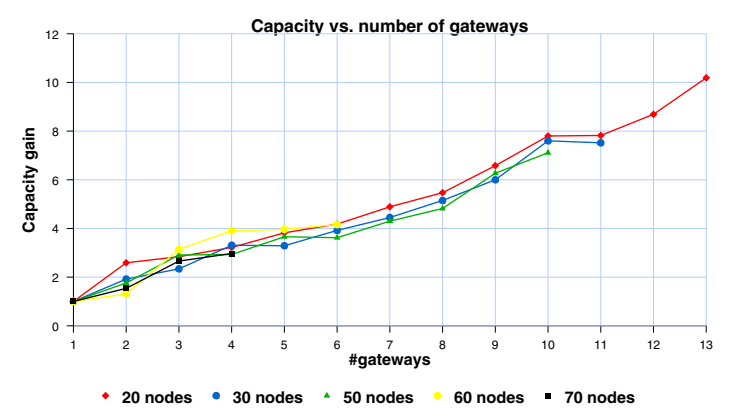

Fig. 4. Capacity increases sub-linearly as the number of gateways grows. (Capacity is normalized by its value with 1 gateway) generic with respect to interference models. We have developed a column generation process that is efficient enough to compute the transport capacity of large scale networks.

We have validated this process on many instances with a classical interference model. Simulations give insights on two key issues of WMN design. We conjecture that computing an optimal routing and scheduling for gathering traffic on a set of gateways can be done in polynomial time, even with a distributed protocol. We also conjecture that one can define the thickness of gateway, a minimal interspace which is necessary and sufficient for the gateway to gather traffic optimally.

These conjectures would yield very efficient WMN design algorithms and flexible traffic management protocols.

\section{ACKNOWLEDGMENTS}

C. Molle PhD is funded by DGA, France. This work has been partially funded by european projects IST/FET AEOLUS and COST 293, ANR-JC OSERA, and ARC CARMA.

\section{REFERENCES}

[1] I. Akyildiz, X. Wang, and W. Wang, "Wireless mesh networks: a survey," Computer Networks, vol. 47, no. 4, pp. 445-487, Mar. 2005.

[2] K. Jain, J. Padhye, V. Padhamanabhan, and L. Qiu, "Impact of interference on multi-hop wireless network performance," in ACM MobiCom, Sep. 2003, pp. 66-80.

[3] M. Kodialam and T. Nandagopal, "On the capacity region of multi-radio multi-channel wireless mesh networks," in IEEE WiMesh, Sep. 2005.

[4] P. Gupta and P. Kumar, "The capacity of wireless networks," IEEE Trans. Inf. Theory, vol. 46, no. 2, pp. 388-404, Mar. 2000.

[5] O. Dousse, M. Franceschetti, D. Tse, and P. Thiran, "Closing the gap in the capacity of random wireless networks," in IEEE ISIT, Jun. 2004.

[6] H. Rivano, F. Theoleyre, and F. Valois, "Capacity evaluation framework and validation of self-organized routing schemes," in IEEE IWWAN, vol. 3, Sep. 2006, pp. 779-785.

[7] J. Jun and M. Sichitiu, "The nominal capacity of wireless mesh networks," IEEE Wireless Commun. Mag., vol. 10, no. 5, pp. 8-14, Oct. 2003.

[8] H. Liu and B. Zhao, "Optimal scheduling for link assignment in trafficsensitive stdma wireless ad-hoc networks," in ICCNMC, ser. LNCS, vol. 3619, Aug. 2005, pp. 218-228.

[9] P. Wang and S. Bohacek, "Toward tractable computation of the capacity of multihop wireless networks," in IEEE INFOCOM, May 2007, pp. 2099-2107.

[10] R. Klasing, N. Morales, and S. Perennes, "On the complexity of bandwidth allocation in radio networks with steady traffic demands," Theoretical Computer Science, 2008, to be published.

[11] M. E. Lubbecke and J. Desrosiers, "Selected topics in column generation," Operations Research, vol. 53, no. 6, pp. 1007-1023, Dec. 2005.

[12] J. Zhang, H. Wu, Q. Zhang, and B. Li, "Joint routing and scheduling in multi-radio multi-channel multi-hop wireless networks," in IEEE BROADNETS, Oct. 2005, pp. 678-687.

[13] G. Carello, I. Filippini, S. Gualandi, and F. Malucelli, "Scheduling and routing in wireless multi-hop networks by column generation," in INOC, Apr. 2007.

[14] W.-H. Tam and Y.-C. Tseng, "Joint multi-channel link layer and multipath routing design for wireless mesh networks," in IEEE INFOCOM, May 2007, pp. 2081-2089.

[15] S. Lakshmanan, K. Sundaresan, and R. Sivakumar, "On multi-gateway association in wireless mesh networks," in IEEE WiMesh, Sep. 2006, pp. 135-137.

[16] A. Kumar, M. Marathe, S. Parthasarathy, and A. Srinivasan, "Algorithmic aspects of capacity in wireless networks," in ACM SIGMETRICS, vol. 33, Jun. 2005, pp. 133-144.

[17] C. Gomes and H. Rivano, "Fair joint routing and scheduling problem in wireless mesh networks," INRIA, Tech. Rep. 6198, May 2007.

[18] M. Grotschel, L. Lovasz, and A. Schrijver, "The ellipsoid method and its consequences in combinatorial optimization," Combinatorica, vol. 1, no. 2, pp. 169-197, Jun. 1981. 